\title{
Une approche sémio-didactique de l'activité de l'enseignant de langue en ligne : réflexions méthodologiques
}

A semio-didactic approach of the online language teacher's activity: methodological reflections

Nicolas Guichon

URL : http://journals.openedition.org/educationdidactique/1679

DOI : 10.4000/educationdidactique.1679

ISSN : 2111-4838

\section{Éditeur}

Presses universitaires de Rennes

\section{Édition imprimée}

Date de publication : 7 février 2013

Pagination : 101-116

ISBN : 978-2-7535-2261-9

ISSN : 1956-3485

\section{Référence électronique}

Nicolas Guichon, « Une approche sémio-didactique de l'activité de l'enseignant de langue en ligne: réflexions méthodologiques », Éducation et didactique [En ligne], 7-1 | 2013, mis en ligne le 31 janvier 2015, consulté le 19 avril 2019. URL : http://journals.openedition.org/educationdidactique/1679 ; DOI 10.4000/educationdidactique. 1679 


\title{
UNE APPROCHE SÉMIO-DIDACTIQUE DE LACTIVITÉ DE LENSEIGNANT DE LANGUE EN LIGNE : RÉFLEXIONS MÉTHODOLOGIQUES
}

\author{
Nicolas Guichon, ICAR, Université Lyon 2
}

Cette contribution établit la synthèse méthodologique et épistémologique d'un projet de recherche longitudinal et interdisciplinaire au cours duquel des chercheurs en didactique des langues et en informatique ont cherché à appréhender l'activité d'enseignement de langue en ligne synchrone et à proposer des outils pour faciliter l'activité de médiation et de développement professionnel des enseignants. La recherche s'appuie sur une formation dans laquelle des apprentis sont placés en situation d'enseigner le français langue étrangère à des apprenants distants par le biais d'une plateforme de visioconférence. Les moyens méthodologiques pour saisir les facettes de l'activité d'enseignement en ligne sont explicités et, selon la démarche sémio-didactique adoptée, plusieurs aspects liés à la conduite de l'interaction en ligne et au développement d'une conscience sémio-pédagogique critique sont présentés.

Mots-clés : enseignement de langue en ligne, transcription multimodale, multimodalité, conscience sémio-pédagogique critique

\section{A semio-didactic approach of the online language teacher's activity : methodological reflections}

This contribution assesses from methodological and epistemological perspectives a five-year interdisciplinary project whereby researchers in language education and in computer science have endeavoured (1) to study online synchronous language teaching, and (2) to develop tools to support the teachers' mediation activity and foster their professional development. The project concentrates on a training situation in which trainees had to teach French as a foreign language to distant learners via a web-conferencing platform. The methods that have been used to study the different facets of the online teachers' activity are detailed. The rationale for a semio-didactic approach is explained as well and several aspects pertaining to the management of the online interaction and the development of a critical semiotic awareness are presented.

Keywords: online language teaching, multimodal transcription, multimodality, critical semio-pedagogical awareness 
Nicolas Guichon

\section{INTRODUCTION}

L'étude de l'activité professorale des enseignants de langue présente des enjeux méthodologiques et conceptuels cruciaux pour les chercheurs en didactique : quand l'approche ethnographique est favorisée, il s'agit d'analyser de quelles façons les membres d'une classe de langue communiquent et participent à la création d'un contexte commun (Cambra Giné, 2003). D'autres chercheurs abordent la classe langue par le biais des interactions et de l'examen du répertoire didactique des enseignants (Cicurel, 2011). Le présent article se situe dans cette double lignée épistémologique et présente un certain nombre de concepts et de méthodologies déployés dans le cadre d'un projet de recherche interdisciplinaire avec des chercheurs en sciences du langage, en informatique et en psychologie cognitive. Ce projet comportait deux visées principales : (1) investiguer le tutorat de langue en ligne synchrone du point de vue des enseignants et (2) concevoir un dispositif (Visu) dédié à l'apprentissage vidéographique synchrone et à la récupération de traces visant le développement professionnel par le biais de la confrontation des apprentis aux traces de leur activité (Bétrancourt, Guichon \& Prié, 2011).

Ce projet de recherche, qui s'est déroulé entre 2006 et $2011^{1}$, s'appuyait sur la situation suivante : des apprentis enseignants en master professionnel de français langue étrangère (FLE) à Lyon 2 participent à un module de formation pendant lequel ils préparent sept séances pédagogiques pour des étudiants étatsuniens (UC Berkeley), les animent lors d'interactions de 45 minutes, puis participent à des débriefings afin de développer des compétences professionnelles relatives à l'enseignement de langue en ligne (Develotte, Guichon \& Kern, 2008 ; Guichon \& Drissi, 2008 ; Guichon, 2009).

En raison de la médiatisation de l'activité d'enseignement par les technologies (en particulier la webcam, le microphone et l'outil de clavardage), l'approche adoptée pour étudier cette situation se concentre sur ses aspects multimodaux et vise à mettre au jour les différentes facettes de l'activité sémio-pédagogique de l'enseignant. Cette dimension sémio-pédagogique de l'activité a trait à l'utilisation appropriée des ressources sémiotiques et technologiques pour favoriser l'apprentissage. Elle semble cruciale pour l'enseignant en ligne car ce dernier est conduit à reconfigurer, de manière inédite, son acti- vité pour l'ajuster à un environnement technologique particulier et à utiliser et à combiner des outils pour conduire l'interaction avec les apprenants distants. Sont donc en jeu (1) des contraintes liées à la tâche et à la médiatisation (environnement technologique et distance) qui désorganisent et réorganisent l'activité, (2) des ressources sémiotiques et technologiques et (3) des stratégies, des intentions et un système de valeurs pédagogiques, un ensemble dont le projet a contribué à dénouer le dense écheveau.

Le présent article s'appuie sur l'examen rétrospectif d'un projet de recherche qui, à partir d'une situation de formation, s'est donné les moyens méthodologiques pour définir des unités d'analyse pertinentes afin d'enrichir la compréhension de la formation des enseignants en ligne, et pour participer à la spécification d'un dispositif d'apprentissage médiatisé.

\section{ENJEUX MÉTHODOLOGIQUES}

\section{Les angles d'analyse}

En comparaison avec une situation de face-à-face, l'enseignement en ligne comporte une part cruciale de médiatisation par les technologies, lesquelles modifient profondément les interactions pédagogiques ainsi que les types de médiations que les enseignants mettent en place pour favoriser l'apprentissage. À l'interface entre les protagonistes (enseignants et apprenants), l'écran de l'ordinateur intervient comme une source plus ou moins riche d'informations (écriture dans le clavardage, documents divers), elles-mêmes enrichies par la webcam. Cette dernière fournit un accès partiel, imparfait mais déterminant à l'image et à la voix des interlocuteurs, deux éléments cruciaux dans une situation didactique où la langue seconde est à la fois l'outil pour communiquer et l'objectif principal de l'apprentissage.

L'image fournie par la webcam est composée d'un plan fixe, sans montage, au débit relativement saccadé en deçà des habituelles vingt-quatre images par seconde. Ce plan fixe présente un contenu informationnel assez pauvre dont les éléments les plus remarquables sont :

(1) les micro-événements qui traversent le visage des interlocuteurs (sourires, moues, froncement des sourcils ou du front, etc.) ; 
(2) le mouvement des lèvres qui accompagnent les paroles prononcées par l'interlocuteur avec un décalage ;

(3) quelques éléments du contexte dans lequel se trouve l'interlocuteur (vêtements, décoration, etc.). Toutefois, en raison du cadrage serré et de la fixité du plan, les éléments non verbaux sont dans l'ensemble peu saillants.

En raison de la pauvreté relative de cette image, on pourrait se demander pourquoi, pour les séances d'enseignement en ligne étudiées pendant cinq ans, allumer la webcam crée un événement toujours important pour les apprenants et les apprentis enseignants ; cela constitue une sorte de fête visuelle qui signale le début véritable de l'échange, même lorsque les autres canaux (écrit du clavardage, voix) ont été préalablement activés - et l'image produite par la webcam concentre les regards jusqu'à la fin de l'échange.

En raison de la place centrale tenue par la webcam autour de laquelle s'organise l'interaction pédagogique en ligne, je vais emprunter trois notions à l'analyse filmique en référence à Deleuze (1983), à savoir le champ, le contre-champ et le hors-champ, puis je proposerai la notion de méta-champ. La figure 1 reprend les différents éléments de cette situation d'interaction médiatisée en les schématisant.

Le champ est limité à ce qui est visible à l'écran. C'est un dispositif d'exposition clos. (Deleuze, 1983). Cependant, parce qu'ils sont libres de manipuler la webcam, la clôture du champ dépend du choix des interactants, de leurs stratégies ou leurs préférences conversationnelles. Par exemple, un apprenant de Berkeley (par timidité ou par jeu) choisit une année de ne montrer qu'une partie de son visage, laissant une grande partie de l'écran vide. L'originalité de l'image de la webcam tient en partie à la rareté des indices qu'elle produit, le visage de l'interlocuteur saturant le champ par le biais d'un gros plan fixe. Le visage sur lequel se concentre la webcam semble avoir une valeur métonymique, le visage de l'enseignant devenant par extension celui de la langue et de la culture francophone. Les entretiens menés auprès des étudiants de Berkeley ont souligné combien cette situation leur paraissait authentique ( genuine») par rapport à la situation de face-à-face pédagogique qu'ils connaissaient par ailleurs, dès lors que l'image de leur enseignant était visible ${ }^{2}$.

Figure 1 : la conversation en ligne selon plusieurs points de vue

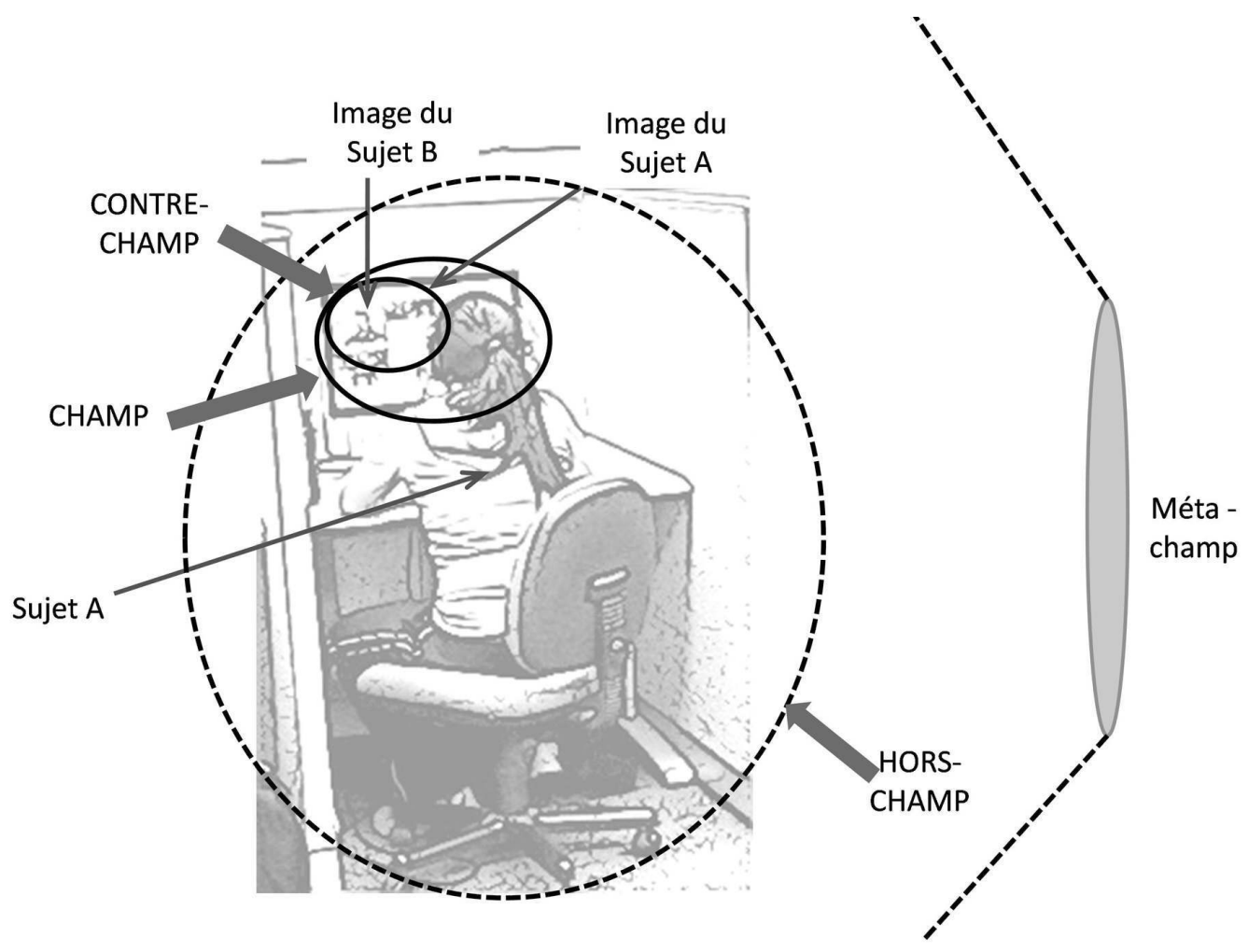


Nicolas Guichon

Le contre-champ est constitué par la propre image des interactants, présente en miniature incrustée dans l'image de l'interlocuteur. Par rapport à une situation ordinaire d'enseignement, le contrechamp permet à chacun de se voir, ce qui offre par exemple la possibilité à l'enseignant en ligne d'autoréguler sa propre image en vérifiant ce qu'il donne à voir à ses apprenants (qualité du cadrage, caractère explicite d'une expression faciale, théâtralisation d'un geste). A priori, cette affordance de l'outil permet de renforcer la dimension empathique de l'échange.

Le hors-champ correspond aux entours de l'interaction, c'est-à-dire tout ce qui dépasse le cadre de l'écran mais participe à l'interaction pédagogique : (1) les éléments physiques du contexte (par exemple les documents et outils dont disposent les interactants) et (2) des éléments symboliques (les représentations de l'autre, de sa langue et sa culture, de sa culture d'apprentissage ou d'enseignement). Le horschamp physique peut parfois faire irruption dans le champ lorsqu'un étudiant va prendre la webcam et balayer la pièce dans laquelle il se trouve à Berkeley. Il intervient également lorsqu'un enseignant opérant de chez lui va devoir s'occuper de son enfant en bas âge et le faire apparaître à l'écran pour le présenter à ses étudiantes, créant un lien entre la situation pédagogique et le contexte social, l'enseignant devenant soudain père. Le hors-champ symbolique est omniprésent mais invisible : il dépend de la construction du contexte commun (voir la notion de grounding ${ }^{3}$ ) qui se négocie entre les interactants en particulier par rapport à des éléments culturels (représentation des valeurs de l'autre, de son contexte socio-économique, des registres de langue appropriés ...). Comme le note Deleuze (1983 : 29), à propos du hors-champ, « un ensemble étant cadré, donc vu, il y a toujours un plus grand ensemble, ou un autre avec lequel le premier en forme un autre ». D'évidence, champ et hors-champ entretiennent un lien consubstantiel, le hors-champ alimentant le champ et faisant qu'une apprentie enseignante aura tel comportement interactionnel plutôt qu'un autre face à ses apprenants distants.

À la différence des trois autres notions, le métachamp ne provient pas du domaine de l'analyse filmique mais de celui de la clinique de l'activité en référence directe aux travaux de Clot (1999; 2008). Je propose de définir le méta-champ comme une vue extérieure et orientée de l'ensemble de son activité sur lequel un sujet en formation peut opérer des sélections et apporter des commentaires et des interprétations afin de faciliter l'analyse critique de son activité. Le méta-champ correspond à la capture de l'échange en ligne par le biais d'un film, d'une capture d'écran dynamique (voir plus haut) ou de tout autre système de traçage, qui permet de fournir une documentation de l'interaction et de la réifier à des fins d'auto-analyse. Bien que réifiée, c'est une vue éminemment subjective (chaque sujet ne distingue pas les mêmes éléments et n'accorde pas la même attention aux mêmes aspects). Elle dépend de la capacité des sujets en formation à créer des liens entre les différents éléments de l'interaction en ligne (champ, contrechamp et hors-champ).

Si l'on résume ces quatre angles, on peut dire que :

- le champ fonctionne comme une fenêtre ouverte (1) sur la langue et la culture francophone si on se place du côté de l'apprenant et (2) sur chaque apprenant envisagé dans son individualité si on se place dans la perspective de l'enseignant ;

- le contrechamp augmente le potentiel interactionnel du dispositif en donnant accès à sa propre image et en permettant certains ajustements ;

- le hors-champ, bien qu'invisible, enrichit subtilement le champ de propriétés symboliques, étire et entrouvre le cadre de l'interaction dans un jeu de relations complexes avec ce qui est donné à voir dans le champ ;

- le méta-champ est une déconstruction de ces trois éléments, une mise à plat désynchronisée de l'activité des enseignants. Celui-ci peut fonctionner comme une ressource pour examiner sa propre activité afin de développer des compétences professionnelles pour les apprentis enseignants et des compétences langagières et interactionnelles pour les apprenants de L2.

Ces quatre notions constituent autant d'angles pour aborder les données empiriques recueillies en vue de l'étude des différents aspects de l'interaction pédagogique médiatisée. Ces données sont principalement de deux ordres :

- le discours sur l'action recueilli par le biais d'entretiens semi-directifs ou d'auto-confrontation, de films de séances de débriefing et de réflexion collective ;

- le discours pendant l'action et les traces de l'activité recueillis par l'intermédiaire des captures d'écran dynamique puis par l'outil de traçage de l'activité de Visu (voir infra). 


\section{Les captures d'écran dynamique}

Étant donné la centralité de cette démarche de recueil de données qui a fourni la majorité du corpus étudié pour ce projet de recherche, il semble important de décrire plus avant ce que les captures d'écran dynamique apportent à la compréhension de l'activité d'enseignement en ligne.

Les logiciels de captures d'écran dynamique ${ }^{4}$ permettent d'obtenir une sorte de film de tous les événements qui se produisent sur l'écran d'ordinateur ; ils ont constitué pour cette recherche l'outil principal pour recueillir la trace des interactions entre les apprentis enseignants et les apprenants (figure 2).
Cette technique de recueil présente plusieurs avantages :

- elle est peu intrusive, les utilisateurs oubliant rapidement qu'un logiciel garde une trace de leur activité $^{5}$, et cette technique ne dénature pas l'activité des sujets comme cela est davantage le cas avec la présence d'une caméra ;

- elle est exhaustive car elle capte tous les événements à l'écran ainsi que toutes les interactions orales, restituant ainsi l'épaisseur de l'interaction avec les éléments temporels et le jeu des différentes modalités (écrit, oral, image fixe et vidéo) ;

- elle donne accès à un texte multimodal ${ }^{6}$, c'est-àdire mettant en jeu une gamme de codes sémiotiques variés qui participent à la construction du sens d'une interaction, et peut ainsi se prêter à l'analyse.

Figure 2 : capture d'écran dynamique

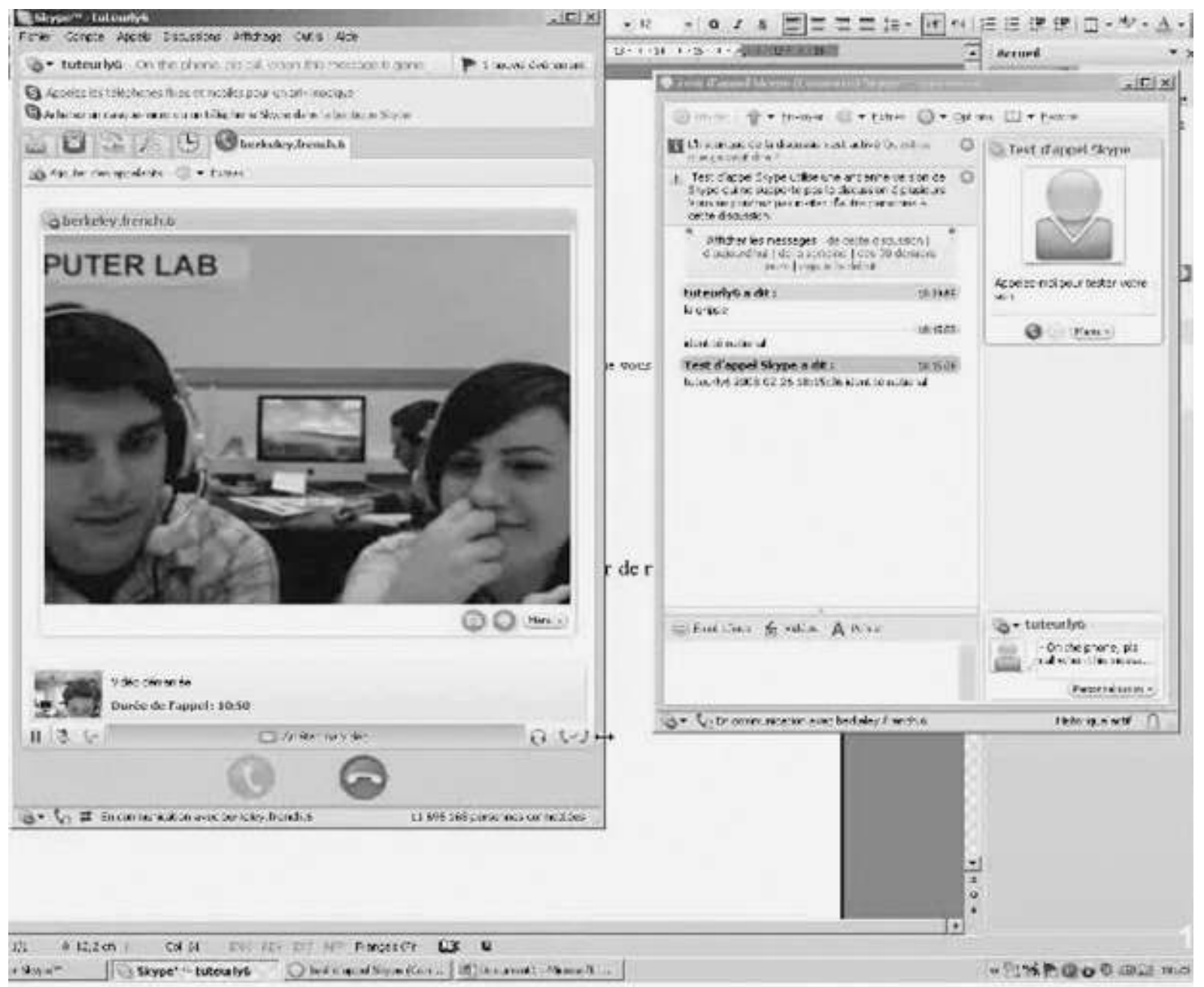


Nicolas Guichon

L'enseignement de langue en ligne se prête bien à cette captation car c'est une activité statique (les participants sont devant leur ordinateur) et la réalisation de l'activité est principalement orale et mimo-gestuelle (gestes et expressions du visage). De manière intéressante, quand il s'agit d'analyser les interactions synchrones en ligne, c'est-à-dire comment et à quel rythme les différentes modalités interviennent au cours de celles-ci, la transcription multimodale (figure 3) décompose les différents éléments de sens afin de montrer comment ceux-ci se combinent ou se dissocient et contribuent à la construction d'une nouvelle grammaire entre les interactants.
Un tel exemple de transcription multimodale, effectuée par Samira Drissi pour une étude menée conjointement (Guichon \& Drissi, 2008) et dans le cadre de son travail doctoral (Drissi, 2011), permet de comprendre comment les différents éléments de l'interaction sont décomposés.

L'échantillon de retranscription multimodale présenté ci-dessus montre, par exemple, de quelle façon différents moyens pour encourager les apprenants sont déployés par les apprenties enseignantes par le biais de la voix, de leurs rires ( $\operatorname{tdp} 115,122$ et 123), de leurs mimiques (tdp 121) et de l'utilisation des émoticônes (tdp 117). L'extrait dévoile également de quelle façon certains des ressources

Figure 3 : exemple de retranscription multimodale

\begin{tabular}{|c|c|c|c|c|c|c|c|}
\hline \multicolumn{2}{|r|}{ TDP } & \multicolumn{3}{|c|}{ TEMPS } & \multirow{2}{*}{$\begin{array}{l}\text { AUDIO } \\
\text { VOCAL }\end{array}$} & \multicolumn{2}{|c|}{ VISUEL } \\
\hline & & $\begin{array}{l}\text { DEBUT } \\
\text { ACTIVITE } \\
\end{array}$ & $\begin{array}{l}\text { FIN } \\
\text { ACTIVITE }\end{array}$ & DUREE & & $\begin{array}{l}\text { FENETRE } \\
\text { CLAVARDAGE } \\
\end{array}$ & WEBCAM \\
\hline 114 & JAI & $14: 04,5$ & $14: 06,0$ & $00: 01,5$ & em elle aime les gâteaux & & \\
\hline 115 & CEL & $14: 06,2$ & $14: 10,0$ & $00: 03.8$ & oui (rires) beaucoup & & \\
\hline 116 & SIL & $14: 10,0$ & $14: 14,2$ & $00: 04,2$ & & & \\
\hline 117 & PAU CLA & $14: 14,2$ & $14: 29,8$ & $00: 15,6$ & & 0 & \\
\hline 118 & CAI & $14: 15,2$ & $14: 19,4$ & $00: 04,2$ & elle a (1.5) les yeux bleus & & \\
\hline 119 & CEL & $14: 20,1$ & $14: 22,2$ & $00: 02,1$ & ouais (0.6) exactement & & \\
\hline 120 & JAI WBC & $14: 29,8$ & $14: 31,5$ & $00: 01.7$ & & & \\
\hline 121 & CAI WBC & $14: 31,5$ & $14: 32,5$ & $00: 01,0$ & & & \\
\hline 122 & PAU & $14: 32,5$ & $14: 36,5$ & $00: 04,0$ & $\begin{array}{l}\text { [rires] (1.4) une dernière } \\
\text { chose }\end{array}$ & & \\
\hline 123 & CEL & $14: 32,5$ & $14: 34,0$ & $00: 01,5$ & $\begin{array}{l}\text { [rires] ( } 2.5) \\
\text { une dernière chose }\end{array}$ & & \\
\hline 124 & PAU CLA & $14: 37,0$ & $14: 40,0$ & $00: 03,0$ & & $\begin{array}{l}\text { une dernière } \\
\text { chose? }\end{array}$ & \\
\hline
\end{tabular}

Légende: tuteur

apprenant 
des enseignants sont repris dans une autre modalité par les apprenants (pouce levé dans le cadre de la webcam, tdp 120 et 121) montrant qu'une grammaire se met en place entre les interactants. Une telle retranscription permet de visualiser les différents composants de ce type d'interaction tout en constituant déjà, par les choix des découpages, un premier niveau d'interprétation.

Il s'agit dès lors d'analyser les interactions selon une méthode inductive, qui, comme le rappelle Traverso (2004: 22), « part des données en cherchant à identifier des comportements interactionnels récurrents, pour en proposer des catégorisations et formuler des généralisations ». L'utilisation des différentes ressources multimodales à disposition et le cadre de l'échange pédagogique à distance apportent deux éléments nouveaux dans l'étude.

\section{LE TRAVAIL D'ANALYSE}

\section{Champ/contre-champ : multimodalité et rythme de l'interaction}

L'étude de l'utilisation de la webcam par les apprentis s'est révélée particulièrement riche étant donnée l'importance sémiotique que celle-ci détient à la fois comme complément au message oral et comme facilitateur de l'intercompréhension (Develotte, Guichon \& Vincent, 2010). Par le biais de la webcam, les échanges en ligne sont enrichis grâce aux expressions faciales ou grâce aux gestes qui remplissent différentes fonctions interactionnelles. Ceux-ci permettent par exemple de coordonner les échanges et leur contenu, de guider le discours (une expression de surprise pouvant par exemple entraîner une reformulation des apprenants), ou d'apporter des encouragements. Ils participent ainsi pleinement à la construction du discours. Toutefois, l'absence de contact direct et la qualité relative de l'image associée aux décalages nécessitent une attention d'autant plus accrue à ces indices qu'ils sont moins accessibles qu'en face-à-face. Les différents entretiens menés auprès des apprentis enseignants et des apprenants confirment que l'utilisation opportune de la webcam crée « un effet de présence à distance » (Weisberg, 1999), installant un terrain commun entre les interactants et améliorant la qualité de la relation pédagogique.

Comme l'ont souligné Kress et Van Leeuwen (1996 : 181), deux alternatives sont possibles pour étudier un corpus multimodal : considérer le sens du texte d'ensemble comme la somme des sens des parties qui le composent ou bien aborder les différentes modalités comme interagissant les unes avec les autres et possédant un impact les unes sur les autres. C'est la seconde approche qui a, par exemple, été choisie pour déterminer de quelle façon la webcam était utilisée par les apprentis enseignants (Develotte, Guichon et Vincent, 2010).

En effet, pour cette étude, nous nous sommes concentrés sur l'image des interlocuteurs pour déterminer différents degrés d'investissement de la webcam (d'un investissement nul, une page de blog recouvrant le visage de l'enseignante, à un investissement maximal, l'enseignante regardant la webcam pour donner l'impression aux apprenants qu'elle les regarde dans les yeux). Le degré le plus élevé correspond d'ailleurs à une sorte de tour de force sémiopédagogique impossible à soutenir dans la longueur car il requiert de l'enseignant de se concentrer sur la diffusion de son regard au détriment de la réception de l'image de son interlocuteur. De Chanay (2011: 150) souligne ainsi le paradoxe de ce type d'échange : «celui qui regarde droit dans les yeux ne voit pas celui qu'il regarde, et celui qui est regardé ne rend pas la pareille ». De Chanay explique l'inconfort des conversations en ligne par le fait que « les participants regardent très peu la caméra, et que lorsqu'ils le font, c'est très furtivement puisque le gain de contact qu'ils essaient de donner à l'autre s'accompagne

Figure 4 : cinq degrés d'investissement de la webcam par les apprentis enseignants

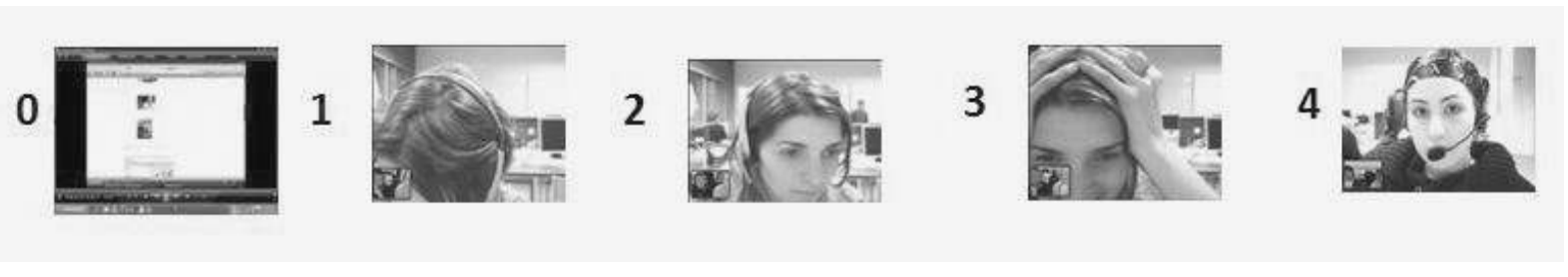


immédiatement, de leur propre côté, d'une perte totale de contact visuel » (ibid.).

Enfin, il faut souligner que le caractère quasisynchrone de l'interaction ajoute un paramètre crucial dans l'étude de la multimodalité : le rythme avec lequel les modalités sont associées ou dissociées, les silences, les pauses et les micro-coupures de la connexion sont autant de phénomènes à prendre en compte pour restituer l'ensemble de l'interaction.

L'étude du système d'alternance des tours (Dejean-Thircuir, Guichon \& Nicolaev, 2010) nous a ainsi permis d'observer la gestion du rythme de l'interaction et l'ajustement des prises de parole entre les apprenants. L'extrait suivant met au jour les nombreuses hésitations et les pauses silencieuses de la part de l'apprenante qui est ici interrogée sur ce qu'elle imagine être les métiers typiques des Français. La gestion des tours de parole par l'enseignant témoigne de son effort de ne pas interrompre la conversation pour laisser à l'apprenante le loisir de construire son intervention.

1. ENSEIGNANT et euh quel métier il ferait le français dans ton imagination

2. APPRENANT quel métier/

3. ENSEIGNANT oui métier

4. APPRENANT oui euh (1.5) les Français sont (3.4) les : : : (1.0) les métiers c'est euh oui il fait les métiers comme (1.9) hum : : (3.4) comme un (3.4) euh couturier de vêtements (1.0) et comme (2.2) les euh vendeurs ou vendeuses de la nourriture dans la rue (1.6) et euh (1.2) euh (1.8) euh : :(1.3) c'est euh (1.6) il travaille dans le métro (1.5) et aussi il (3.8) il fait la cuisinE et : : (4.4) oui c'est je ne peux pas pense aux autres

Grâce à cet examen, il est ainsi apparu que certains enseignants en ligne adoptent des conduites langagières et saturent les silences qui ponctuent les tours de parole en reformulant leurs propres énoncés, limitant ainsi les possibilités d'expression et de déploiement de leurs compétences discursives par les apprenants. Le caractère synchrone de l'enseignement en ligne nous a donc incités à étudier minutieusement l'impact de la synchronie sur la gestion du rythme des tours de parole et sur les phénomènes de chevauchements, de silences et de pauses. Ainsi, non seulement les enseignants sont amenés à opérer des choix parmi les différentes modalités dont ils disposent dans leur environnement de travail, mais, il leur échoit également de séquencer l'information et d'adapter le rythme de leurs énoncés aux besoins et aux niveaux de leurs apprenants distants en prenant en compte les particularités de cette communication (débit ralenti, désynchronisation de la voix et du mouvement des lèvres). Multimodalité et rythme de l'interaction sont donc intimement liés dans ce type d'échange synchrone.

\section{Le hors-champ de l'interaction pédagogique en ligne}

Malgré l'intérêt des données obtenues par le biais des captures d'écran dynamique, certains angles morts de l'interaction se sont fait jour. En effet, l'ensemble de l'interaction à distance ne se joue pas que sur l'écran de l'ordinateur mais une partie déborde de ce cadre et, pour reprendre une notion provenant de l'analyse filmique évoquée plus haut, crée un hors-champ par rapport à l'interaction médiatisée elle-même.

Figure 5 :

une apprentie enseignante au travail vue de profil

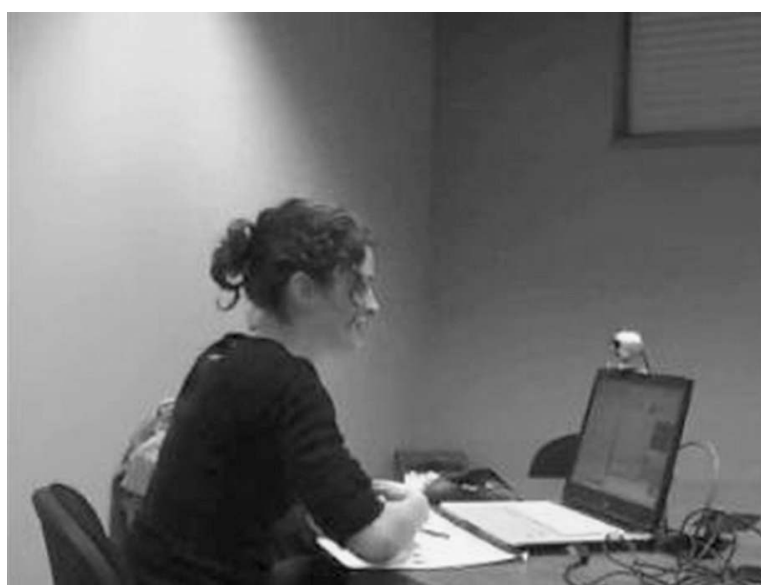

Ainsi, d'autres techniques de recueil de données ont été mises en place (figure 5 montrant le filmage en plan de coupe d'une enseignante au travail) pour compléter l'analyse de l'activité en donnant à voir comment les apprentis organisent leur travail, quels outils (crayon, papier, etc.) non visibles dans le cadre de l'écran participent cependant à la construction de l'interaction. Une telle approche ménage une perspective depuis « les coulisses de l'activité » (Cicurel, 2007) et permet par exemple d'appréhender certains phénomènes liés à la polyfocalité de l'attention 
(Scollon, Bhatia, Li \& Yung, 1999 : 35), c'est-à-dire la façon que l'apprenti enseignant a de gérer différents outils, différents plans de travail et différents objectifs de manière quasi simultanée.

La compréhension des difficultés rencontrées par les apprentis tuteurs était en effet cruciale pour nourrir le travail de spécification de la plateforme Visu qui était l'objectif d'application du projet de recherche (voir l'introduction). Comme Hoc, Amalberti, Cellier et Grosjean (2004 : 39) l'ont souligné, quand la pression temporelle s'ajoute à la nouveauté d'une situation, il est probable que les difficultés s'accroissent puisque les contraintes qui pèsent sur les capacités attentionnelles des individus sont importantes et leurs capacités à résoudre les problèmes sont ralenties. En suivant les propositions de Clot (1999 : 101) d'approfondir l'analyse d'une situation de travail en examinant les difficultés rencontrées dans le cours de l'action, cette perspective depuis le hors-champ de l'activité nous a permis de proposer un certain nombre de fonctionnalités et de recommandations pour faciliter le travail des enseignants en ligne (Guichon, 2010).

La plupart des difficultés identifiées grâce à cette approche ont trait aux interactions entre les enseignants et leurs apprenants distants (par exemple la difficulté de savoir s'ils ont reçu on non un document) ainsi qu'à la gestion de différents outils tandis qu'ils sont engagés dans diverses tâches. Cette polyfocalité de l'attention est typique du travail enseignant mais la médiatisation de l'interaction et la confrontation des apprentis avec des outils, des fonctionnalités et des interfaces relativement nouveaux a sensibilisé l'équipe des chercheurs du projet aux limites attentionnelles des sujets humains en ce qui concerne la gestion des ressources cognitives et les contraintes particulières de la tâche "enseigner en ligne de manière synchrone avec des outils de visioconférence ».

Un autre type de hors-champ est celui fourni par la captation des sessions de débriefing qui confrontent les apprentis enseignants à des échantillons de leur performance par le biais des captures d'écran dynamique (figure 6). Les commentaires et les analyses suscitées permettent cette fois de comprendre les intentions des enseignants, leurs représentations de leur activité, les objectifs qu'ils se sont fixés, mais aussi les émotions que l'activité ou le visionnage de cette activité suscitent (Guichon, 2009 et 2011). Confronter les participants à l'image de leur activité (et à l'analyse des chercheurs par rapport à cette activité) semble particulièrement propice pour assurer

Figure 6 : visionnage des captures d'écran dynamique lors des sessions de debriefing

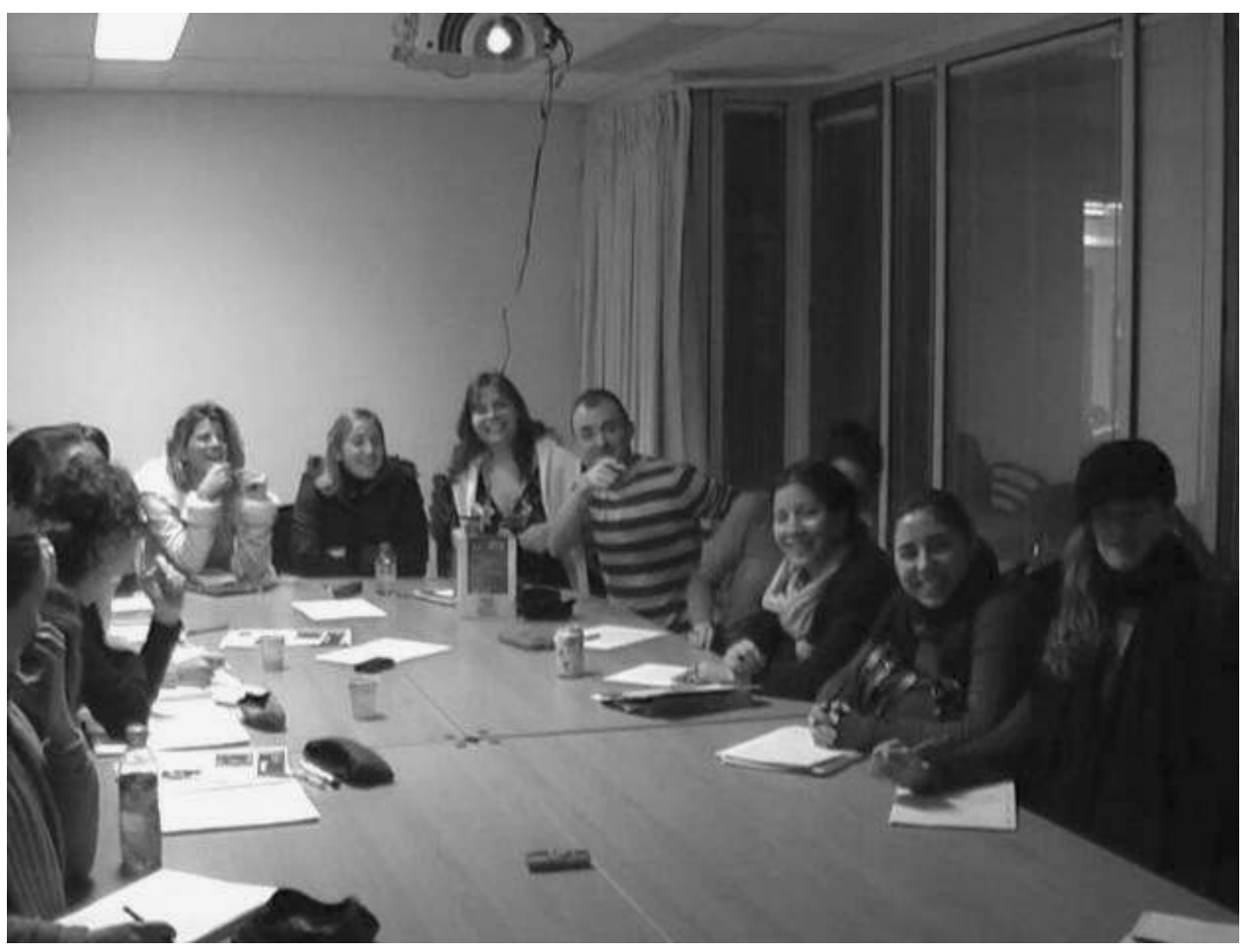


Nicolas Guichon

la validité interprétative de l'analyse, une visée scientifique cruciale selon Dörnyei (2007 : 58). Que ce soit lors de sessions de débriefing collectives ou d'autoconfrontation, c'est cette validité interprétative qui a été recherchée à de nombreuses reprises pour s'assurer que les futurs utilisateurs du dispositif d'apprentissage Visu étaient associés à la démarche de conception aussi étroitement que possible. L'objectif visait à augmenter l'utilité des procédures de formation et l'utilisabilité des outils de communication et d'enseignement selon une démarche de conception continuée par l'usage (Rabardel et Pastré, 2005 : 3).

Dans la même lignée que l'autoconfrontation, mais plus loin de l'activité elle-même, les différents entretiens réalisés auprès des apprentis enseignants et des apprenants permettent d'affiner les perceptions des uns et des autres par rapport à l'ensemble des éléments (programmes, formateurs, indications de lieu) qui, bien que n'étant pas inclus dans le champ direct de l'interaction en ligne, lui sont néanmoins rattachés de façon symbolique.

Figure 7 : dessin d'une apprenante de Berkeley (2010)

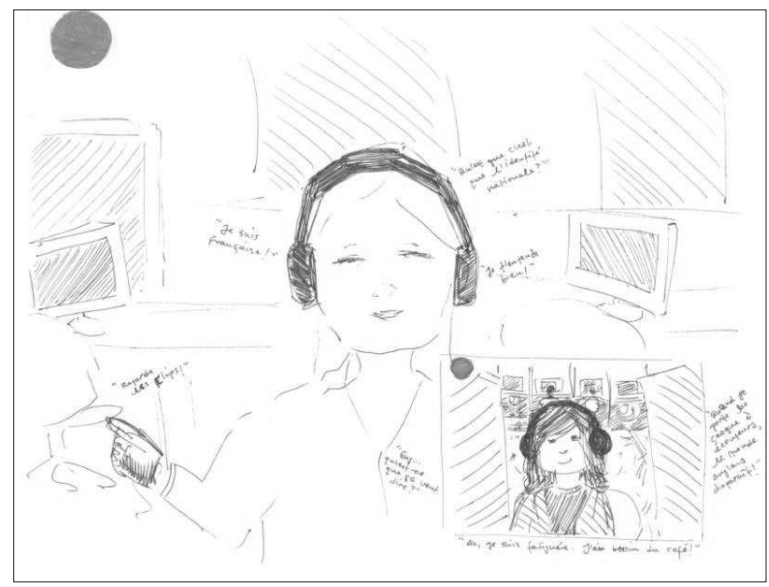

Ainsi, chaque année, il a été demandé aux étudiants de Berkeley de proposer une représentation de leur expérience de l'interaction par le biais d'un dessin. Comme le rappelle Sallaberry (2003: 7), « une représentation est toujours à la fois une représentation d'un objet par un sujet, mais aussi une représentation du sujet. Ce dernier, en effet, en communiquant, donc en décrivant comme il se représente le monde, les choses, les gens, se dévoile». Les choix qui sont faits pour représenter l'interaction donnent des indications quant à ce qui oriente la subjectivité des protagonistes. Par exemple, dans le dessin d'une étudiante de Berkeley présenté à la figure 7 , la présence de multiples écrans d'ordinateurs, les points rouges signalant que l'interaction est enregistrée, le sourire bienveillant de l'enseignante. Ces éléments de surface peuvent ensuite être utilisés pour conduire un entretien semi-directif et enrichir la description de la situation.

Enfin, d'autres données peuvent être provoquées par le biais d'entretiens, comme cela apparaît dans les deux extraits présentés ci-dessous, enrichissant la compréhension de l'interaction entre chacun des protagonistes et permettant par exemple d'aborder les aspects communicationnels (le passage abrupt d'un sujet à un autre pour l'apprenante, la difficulté à gérer les chevauchements dans l'interaction pour l'apprentie) ou interculturels.

Tableau 1 : entretien d'une étudiante de Berkeley au sujet de son expérience

SP : We'd generally, like, say hello and ask each other like how was your week and talk a little bit about what we did. And then she [the teacher] would start the session and um, give me a picture or a, like short video or something to look at, and um...then she'd ask me questions about, you know, "What does it make you think of," um..."Do you see anything similar to this in the United States?" or "Do you think," like, for the last one I think that there were movie um...uh, trailers and she said "Do you think a movie like this if made in English would succeed in America?" And then I'd, uh, sort of give her my impression of whatever she had sent and, um...she'd sort of move the topic of conversation in another direction by sending, like, something related to it, or um...sort of asking me a question on a slightly different topic related, and it was sort of...um, we'd run out of time occasionally on one topic, so we'd sort of abruptly switch to the next one, and we usually didn't talk about everything that she had to talk about, but...uh

Tableau 2 : fragment d'entretien d'autoconfrontation d'une enseignante

WEN : La grosse différence c'est qu'ils ne sont pas là, enfin y a rien à voir et en même temps des fois je me dis que on peut oublier. La grosse différence, c'est que c'est nettement moins naturel. Par exemple moi ça peut m'arriver de préparer une activité avec des questions. Et de faire complètement autre chose. Et que c'est moins naturel, parce que je suis moins naturelle et qu'elles sont moins naturelles. Je pense que si je les avais en cours en présentiel... Elles pourraient plus facilement rebondir sur plein de trucs. Là, y a un décalage. Du coup, je commence à parler, elle s'arrête de parler et elle croit que je les ai coupées et on est coupé. 
L'étude des interactions en ligne peut donc être complétée en prenant en compte non seulement ce qui est visible au point d'interface entre les interactants, c'est-à-dire l'écran, mais en variant les points de vue afin de restituer ce qui constitue les entours de l'activité. Ces entours comprennent les valeurs (socio-historiques, éducatives, culturelles) des protagonistes de l'interaction mais aussi les contraintes de l'environnement (institutionnelles, matérielles, techniques) qui créent une écologie particulière ${ }^{7}$ autour de l'événement pédagogique lui-même.

Si la médiatisation engendre un aplatissement de l'interaction pédagogique (tout est en deux dimensions dans le cadre de l'écran d'ordinateur), la prise en compte du hors-champ dans l'analyse permet de restituer une partie de la profondeur émotionnelle, symbolique et cognitive qui est en jeu entre les interactants.

\section{FORMER À PARTIR DE L'ANALYSE DE L'ACTIVITÉ : LA RÉTROSPECTION}

Nous avons vu que le méta-champ correspond à une vue de l'activité d'enseignement offrant la possibilité de mettre en interaction tout ce qui passe à l'écran (champ/contrechamp) et autour de l'écran (hors-champ). Il prend toute sa valeur à travers le discours des apprentis lorsque ceux-ci sont confrontés à leur activité et mènent une analyse critique de cette activité. En étant archivé, le film de l'activité située peut ainsi devenir un corpus pour la formation. Les captures d'écran dynamique ont ainsi fourni, dans un premier temps du projet, un accès extérieur à l'activité qui a été utilisé pour nourrir les séances de débriefing suite aux séances d'enseignement. Ainsi, l'activité peut être extraite de l'urgence de la situation, caractéristique de l'activité d'enseignement, et se muer en un objet manipulable à loisir (Guichon, 2011).

L'image de l'activité devient elle-même une technologie qui médiatise le rapport de l'apprenti à une pratique professionnelle en construction ${ }^{8}$. Les outils de l'analyse du discours ont fourni des moyens pour examiner certaines pratiques discursives déployées par les apprentis pour évaluer leur activité et celle de leurs pairs et pour développer un répertoire qui dessine les contours d'un métier émergent (Guichon, 2011). L'analyse du corpus a aussi fait apparaître de quelle façon se joue « la dynamique sociale des apprentissages » (Nonnon, 2008: 43) en mettant au jour de quelles façons les apprentis évaluent leur propre activité et celle de leurs pairs. Non seulement la rétrospection médiatise l'activité de l'apprenti mais elle la socialise car, en étant le lieu de l'autoévaluation et de l'hétéro-évaluation, elle intègre peu à peu l'apprenti dans une communauté professionnelle qui « se sédimente dans le patrimoine collectif de gestes et de mots à faire ou à ne pas faire, à dire ou à ne pas dire, de techniques du corps et de l'esprit » (Clot, 2008: 254).

La figure 8 s'emploie à montrer les différentes facettes de l'activité quand celle-ci fait l'objet d'un travail de rétrospection. J'ai proposé de définir la rétrospection comme l'analyse critique de l'activité par le biais de la confrontation d'un collectif d'individus en formation (dans ce cas d'espèce un groupe d'apprentis enseignants) à des traces de leur activité en situation de travail (telles que livrées par les captures d'écran dynamique ou par le salon de rétrospection de Visu). Ce schéma s'inspire de la modélisation de l'activité par Leplat (1997 : 16) mais ajoute les éléments liés au travail de rétrospection tels qu'ils ont été mis au jour par les différentes analyses du travail des apprentis.

Ainsi, la rétrospection fournit l'opportunité de mettre en relief les compétences professionnelles par le biais de ce que je propose d'appeler une conscience sémio-pédagogique critique. Grâce à l'accès à leur propre activité en situation de travail, l'occasion est donnée aux apprentis enseignants d'apprendre à ajuster les outils à leurs objectifs pédagogiques et à développer une conscience de l'image qu'ils projettent par le biais de la webcam et de l'effet que cette image a sur les apprentissages, mais aussi des autres ressources sémiotiques à leur disposition.

Notons toutefois, à la suite de Leplat (1997), que ce qui est réalisé pour l'acteur de l'activité diffère de ce qui est réalisé pour ses pairs ou pour les experts. Enfin, il serait illusoire de penser que les acteurs (ou les observateurs) peuvent obtenir un panorama complet de l'activité. La rétrospection conserve un certain nombre d'angles morts qu'il convient de préserver pour éviter que le dispositif de rétrospection ne devienne un panoptique révélant toutes les facettes de l'activité, même celles que les acteurs ne sont pas encore prêts à affronter, ce qui pourrait alors les mettre en danger vis-à-vis d'eux-mêmes ou de leurs pairs. 
UNE APPROCHE SÉMIO-DIDACTIQUE DE L'ACTIVITÉ DE L'ENSEIGNANT DE LANGUE EN LIGNE...

Nicolas Guichon

Figure 8 : les facettes de l'activité mises au jour par la rétrospection

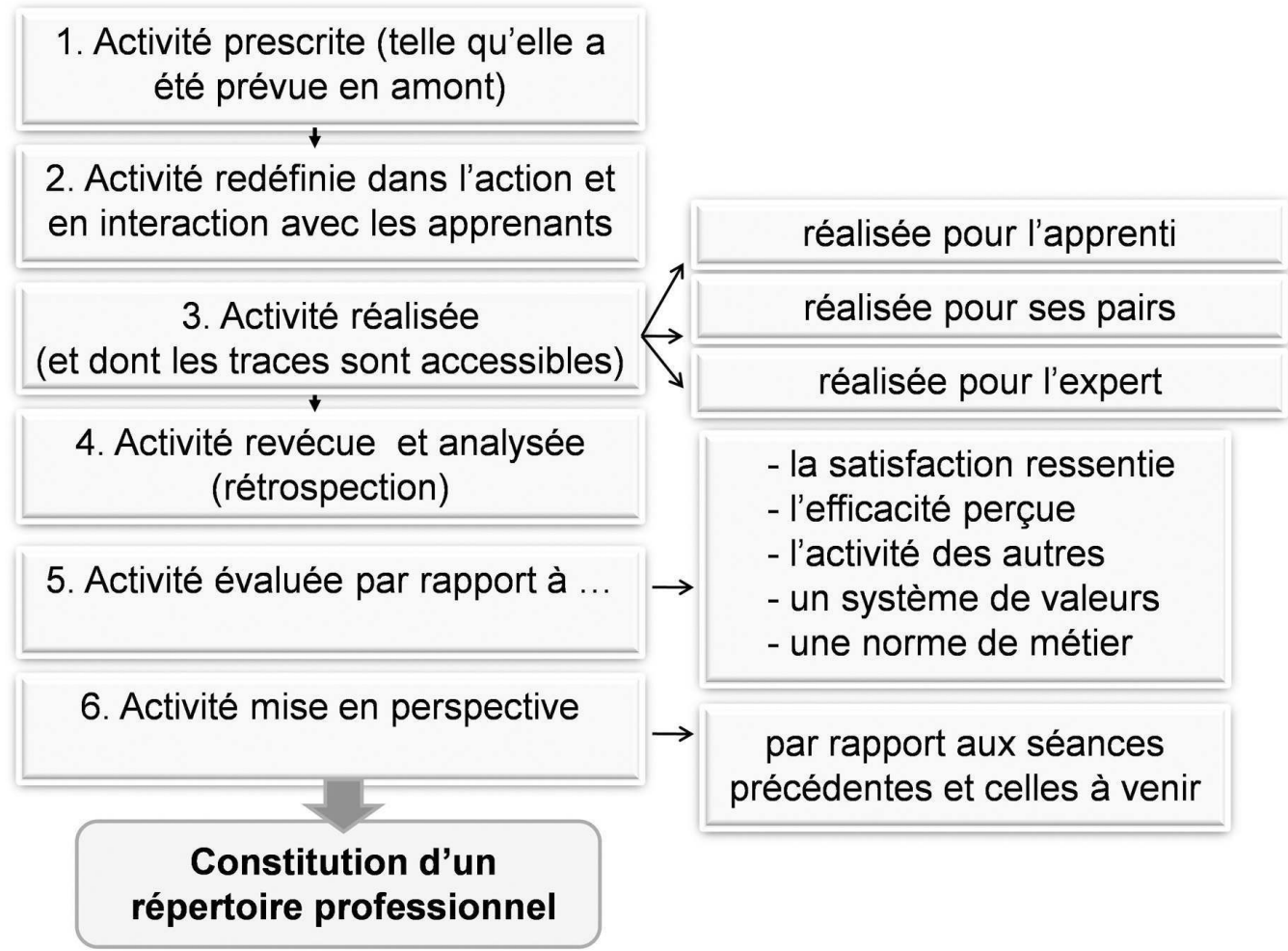

Ce schéma cherche à mettre en lumière que :

- l'activité est un objet protéiforme qui n'a pas la même signification selon qui la regarde et qui en est responsable ;

- elle se dérobe en partie à l'analyse parce qu'elle est travaillée par des valeurs et des émotions ;

- les ressources discursives et sémiotiques dont disposent les apprentis ne peuvent qu'imparfaitement en restituer l'épaisseur et le sens ;

- elle peut néanmoins faire l'objet d'évaluations et d'auto-évaluations qui vont l'étalonner progressivement pour en éprouver l'efficacité ou la validité ;

- les traces de l'activité peuvent servir d'objet intermédiaire pour la construction d'un collectif constitué autour d'une pratique professionnelle ;

- l'entrée par l'activité permet de donner un accès à la pratique non pas seulement telle qu'elle devrait être (selon des référentiels ou des programmes de formation) mais telle qu'elle entre en résonance avec l'identité en construction, la communauté dans laquelle s'inscrit l'individu et la signification que celui-ci donne à son métier.

INSTRUMENTATION TECHNOLOGIQUE : LE SALON DE RÉTROSPECTION

Le projet ITHACA s'est nourri des résultats des différentes études évoquées ci-avant pour fournir des outils destinés à faciliter le travail de rétrospection par le biais d'un archivage des séances (toutes sont conservées sur le serveur), par un système de marquage qui permet d'identifier des épisodes critiques a priori plus porteurs de sens, et enfin par des outils d'annotation qui permettent de sélectionner des échantillons d'une interaction et de se situer par rapport à sa propre activité grâce aux discours (écrits et individuels, puis oraux et socialisés) des apprentis (Guichon, 2010 ; Bétrancourt, Guichon \& Prié, 2011, Guichon, Bétrancourt \& Prié, 2012). Rappelons que Visu agrège deux salons, l'un synchrone (dans lequel l'enseignant peut interagir avec un ou deux apprenants) et l'autre asynchrone (prévu pour que l'enseignant découpe quelques séquences potentiellement acquisitionnelles que l'apprenant concerné peut rejouer afin de repérer certains écarts dans son interlangue). La copie d'écran (figure 9) donne une idée de l'organisation du salon de rétrospection. 
Figure 9 : le salon de rétrospection de Visu

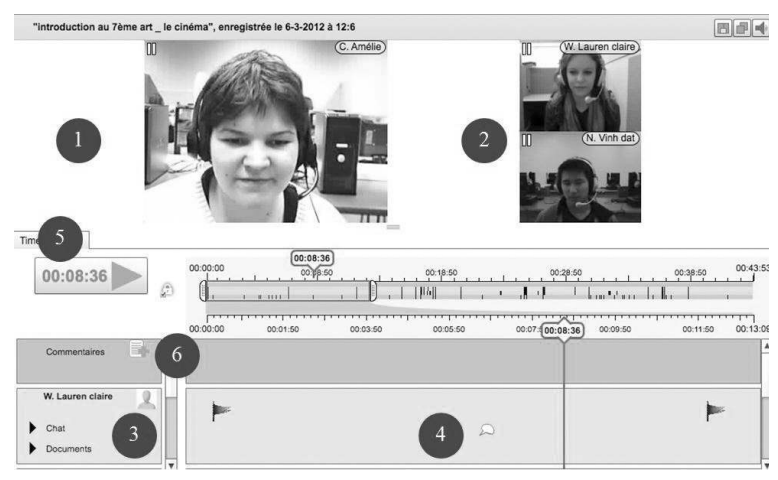

Dans le salon de rétrospection, l'apprenti a accès à sa propre image (1) ainsi que celle des apprenants (2) avec lesquels il a interagi. Chacun des protagonistes de l'interaction pédagogique se voit allouer une ligne (3) qui fonctionne comme une partition musicale, sur laquelle apparaît chacune des actions (4) dont le système a gardé trace, à savoir les consignes et mots-clés enregistrés, les messages écrits dans l'espace clavardage, les documents qui ont été partagés avec les apprenants. Tous ces éléments peuvent être recontextualisés par l'apprenti, qui peut rejouer tout le film de la séance (5) ou bien rechercher certains incidents critiques qu'il a signalés sur la ligne de défilement temporel avec des marqueurs pendant le déroulement de l'interaction pédagogique en ligne (petits drapeaux rouges). En rendant l'activité manipulable (avance et retour rapides, arrêt sur image), on peut ménager une opportunité pour remettre en jeu l'activité réalisée et apprécier le rôle tenu par les protagonistes. Ainsi, avec les diverses fonctionnalités proposées dans le salon de rétrospection, l'enseignant en formation a le loisir de se livrer au même travail d'analyse en annotant les extraits qui lui paraissent dignes d'intérêt (6). Une fois ce travail effectué, il peut partager la trace de son activité ainsi que ses analyses avec ses pairs lors d'un débriefing en présentiel ou à distance. De cette façon, l'image brute de l'activité fait l'objet d'une explicitation progressive qui peut être présentée aux autres membres du collectif d'apprentissage et se transforme ainsi réellement en un corpus pour la formation.

Par rapport aux captures d'écran dynamique, le salon de rétrospection qui a été conçu dans le cadre du projet Visu donne un accès différent de la réalité des interactions. En effet, alors que les captures d'écran dynamique fournissent une vision neutre et globale de l'interaction, le salon de rétrospection décompose l'interaction en différents éléments (le film des protagonistes et le son de leurs voix), les différents objets qu'ils ont manipulés pendant l'interaction (consignes, mots-clés, documents, annotations) qui apparaissent sur un axe temporel. Il faut noter que cette décomposition correspond à l'une des deux approches de la multimodalité repérée par Kress et Van Leeuwen (voir supra). Toutefois, pour utile qu'elle soit pour analyser les différents éléments de l'interaction de manière isolée, cette représentation demande un effort d'imagination pour relier les éléments épars en un tout significatif. Une expérimentation comparant le potentiel de la capture d'écran dynamique et celle du salon de rétrospection pour la formation reste à mener afin de déterminer laquelle des deux représentations présente l'accès le plus riche à la réalité des interactions. On peut supposer que, dans le salon de rétrospection, l'analyse des différents éléments se fait parfois au détriment d'une appréhension de l'interactivité entre les différentes modalités disponibles et de la complexité des rapports que celles-ci entretiennent entre elles. En revanche, par rapport à la capture d'écran dynamique, le salon de rétrospection de Visu permet d'annoter l'interaction et d'ajouter une couche de sens à la réalité de l'interaction. Il fournit aussi des moyens pour arpenter l'interaction à loisir avec des outils de navigation originaux et peut ainsi contribuer à développer la conscience sémio-pédagogique critique des futurs enseignants.

\section{CONCLUSION}

Comme cela apparaît à travers la description de la conception du salon de rétrospection, la démarche de l'équipe de recherche est consonante avec certains travaux en ergonomie et en psychologie (Rabardel, 1995) qui ont enrichi l'approche de l'activité humaine "instrumentée » en dépassant la description des seules affordances du dispositif sociotechnique par l'inclusion de l'examen minutieux de ses usages. Avec ce projet, il s'est agi de prendre en compte non seulement les savoirs et compétences des enseignants, leurs représentations, le contexte de leur activité et de leurs apprentissages, mais aussi les utilisations et détournements des outils mis à leur disposition, et la mobilisation des ressources sémiotiques, cognitives et sociales qui constituent le 
répertoire des enseignants en ligne. Comme le notent Albero et Thibault (2009), cette démarche quand elle est appliquée à la formation ouvre « à une compréhension de l'instrumentation de l'activité en tant que production complexe, dans laquelle interagissent à la fois les données de la technique et les processus de l'appropriation, la fonction pratique mais aussi le projet de l'acteur et le sens qu'il lui attribue ».

Cet article a permis de mettre en lien les différentes études exploratoires qui émaillent une recherche longitudinale autour de la formation et du développement d'un artefact. Pour autant que ces études soient utiles pour éclairer le travail du formateur et du concepteur de dispositifs techno-pédagogiques, il semble indispensable, quand un projet a atteint une certaine maturité de proposer une grille de lecture méthodologique et conceptuelle. Il est à noter que les canaux, champs et temporalités en jeu dans cette interaction ont nécessité d'être décomposés dans un premier temps pour en distinguer la singularité, puis examinés en interaction les uns avec les autres, pour enfin être envisagés en lien avec la propre activité des apprentis enseignants selon la logique anthropo-centrée d'une didactique qui s'intéresse aux technologies non pas pour leurs promesses supposées mais pour les usages développées par les différentes classes d'utilisateurs (Guichon, 2012 : 214-217).

Enfin, c'est en partant des corpus récupérés année après année que les chercheurs associés au projet ont pu graduellement appréhender l'épaisseur de ces interactions pédagogiques particulières par le biais de différentes facettes des activités qui la composent.

\section{NOTES}

1. Cette recherche s'est déroulée dans le cadre du Français en (première) ligne initiée par Christine Develotte, d'un partenariat avec l'UC Berkeley avec Richard Kern, puis d'un projet financé par l'ANR (ITHACA) dont Yannick Prié (Université de Lyon 1) et l'auteur ont assuré la direction scientifique. Je mentionnerai également le travail doctoral très riche de Samira Drissi, Viorica Nicolaev et Caroline Vincent qui ont participé à ce projet et ont largement contribué à en élargir le périmètre scientifique. Enfin, trois développeurs ont particulièrement participé à la conception de Visu : Olivier Aubert, Lionel Breduillieard et Serguei Sayfulin. Cela explique pourquoi bien que la majorité de cet article soit écrit à la première personne du singulier, le pluriel intervient parfois lorsque je fais référence aux dimensions collectives de la recherche.

2. Il est à noter que dans le programme dans lequel s'inscrit cette formation, les étudiants de l'UC Berkeley ont cours de français 5 fois par semaine, le cours avec les apprentis enseignants de Lyon n'intervenant qu'une fois par semaine. Quelques incidents techniques se sont produits chaque année et ont permis, de manière écologique, de vérifier le potentiel de cette interaction médiatisée en mode normal (accès à toutes les modalités) ou en mode dégradé (sans le son ou sans l'image).

3. Selon la psychologie cognitive, « le grounding est le processus à travers lequel les interlocuteurs coordonnent et partagent un ensemble d'informations afin de construire une conversation et la compréhension de son contenu » (Michinov, 2008).

4. Dans ce projet, deux logiciels ont été utilisés : Screen video recorder et Camtasia.

5. L'autorisation écrite a bien entendu été obtenue en amont auprès de tous les participants.

6. «Any text whose meanings are realized through more than one semiotic code is multimodal » (Kress et Van Leeuwen, 1996: 181).

7. On voit le lien qui peut être fait avec le courant des « workplace studies » dont la contribution est soulignée de cette façon par Luff, Hindmarsh et Heath (2000: 17): "Perhaps the most significant contribution of these workplace studies are the ways in which they are placing tool or artefact mediated conduct at the heart of the analytic agenda, and attempting to reconceptualise technology, and in particular reveal how the use and intelligibility of objects is produced and constituted in and through social action and interaction $»$. 8. Dans la même veine de recherches menées à partir de l'image de l'activité en sciences de l'éducation, on peut signaler, NéoPass@ction, une recherche-formation menée 
entre autres par Luc Ria et Patrick Picard. Cette recherche recourt à des données vidéo d'enseignants dans des situations diverses qui sont organisées sur une plateforme de formation en ligne afin de servir comme ressources pour la formation des enseignants débutants. cf. http://neo.inrp.fr/

\section{RÉFÉRENCES}

Albero, B. et Thibault, F. (2009). La recherche française en sciences humaines et sociales sur les technologies en éducation. Revue française de pédagogie. $\mathrm{n}^{\circ} 169$, pp. 5366.

Bétrancourt, M., Guichon, N. et Prié, Y. (2011). Assessing the use of a Trace-Based Synchronous Tool for distant language tutoring. Proceedings of the 9th International Conference on Computer-Supported Collaborative Learning, vol. 1, pp. 478-485. Hong-Kong, July 2011.

Cambra Giné, M. (2003). Une approche ethnographique de la classe de langue. Paris : Didier.

Cicurel, F. (2007). Lagir professoral, une routine ou une action à haut risque? In Plazaola Giger, I. et Stroumza, K. (dirs.). Paroles de praticiens et description de l'activité. Bruxelles: De Boeck. pp. 15-36.

Cicurel, F. (2011). Les interactions dans l'enseignement des langues. Paris : Didier.

Clot, Y. (1999). La fonction psychologique du travail. Paris : PUF.

Clot, Y. (2008). Travail et pouvoir d'agir. Paris : PUF.

De Chanay, H.C. (2011). La construction de l'ethos dans les conversations en ligne. In Develotte, C., Kern, R. et Lamy, M.-N. (eds.). Décrire la conversation en ligne. Le face à face distanciel. Lyon : ENS Éditions. pp. 145-172.

Dejean-Thircuir, C., Guichon, N., Nicolaev, V. (2010). Compétences interactionnelles des tuteurs dans des échanges vidéographiques synchrones. Distances et savoirs. Vol. 8, n 3. pp. 377-393.

Deleuze, G. (1983). L'image-mouvement. Paris : Éditions de Minuit.

Develotte, C., Guichon, N. et Kern, R. (2008). Allo Berkeley? Ici Lyon... Vous nous voyez bien ? Étude d'un dispositif de formation en ligne synchrone franco-américain à travers les discours de ses usagers. Alsic. vol. 11, $\mathrm{n}^{\circ} 2$, pp. 129-156.

Develotte, C., Guichon, N., Vincent, C. (2010). The use of the webcam for teaching a foreign language in a desktop videoconferencing environment. ReCALL, vol. 23, $\mathrm{n}^{\circ}$ 3, pp. 293-312.

Dörnyei, Z. (2007). Research methods in applied linguistics. Oxford : Oxford University Press.

Drissi, S. (2011) Apprendre à enseigner par visioconférence : étude d'interactions pédagogiques entre futurs enseignants et apprenants de FLE Thèse en Sciences du langage. École Normale Supérieure de Lyon.

Guichon, N. et Drissi, S. (2008). Tutorat de langue par visioconférence : comment former aux régulations pédagogiques, Les Cahiers de l'ACEDLE, vol. 5, $\mathrm{n}^{\circ} 1$. pp. 185-217.
Guichon, N. (2009). Training future language teachers to develop online tutors' competence through reflective analysis. ReCALL 21(2): 166-185 .

Guichon, N. (2010). Preparatory study for the design of a desktop videoconferencing platform for synchronous language teaching. Computer Assisted Language Learning. 23(2): 171-184.

Guichon, N. (2011). Former les futurs enseignants de langue en ligne par le biais de la rétrospection. Alsic. vol. $14, n^{\circ} 1$.

Guichon, N., Bétrancourt, M., Prié, Y. (2012). Managing written and oral negative feedback in a synchronous online teaching situation. Computer assisted language learning. vol. 25, No. 2, pp. 181-197.

Guichon, N. (2012). Vers l'intégration des TIC dans l'enseignement des langues. Paris : Didier.

Hoc, J.-M., Amalberti, R., Cellier, J.-M., \& Grosjean, V. (2004). Adaptation et gestion des

risques en situation dynamique. In J.-M. Hoc \& F. Darses (Eds.), Psychologie ergonomique: tendances actuelles. (pp. 15-48). Paris : Presses Universitaires deFrance.

Kress, G. et Van Leeuwen, T. (1996). Reading Images: The grammar of visual design. New York: Routledge.

Leplat, J. (1997). Regards sur l'activité en situation de travail. Contribution à la psychologie ergonomique. Paris : PUF.

Luff, P., J. Hindmarsh, et C. Heath, (eds.). (2000). Workplace Studies : Recovering Work Practice and Informing System Design. Cambridge : Cambridge University Press.

Michinov, E. (2008). La distance physique et ses effets dans les équipes de travail distribuées : une analyse psychosociale. Le travail Humain, vol. 71, $\mathrm{n}^{\circ} 1$. pp. 1-21.

Nonnon, E. (2008). Tensions et dynamique des interactions dans les échanges scolaires. In Fillietaz, L. et Schubauer-Leoni, M.-L. (eds.). Processus interactionnels et situations éducatives. Bruxelles : De Boeck. pp. 43-65.

Rabardel, P. (1995). Les Hommes et les Technologies - Approche cognitive des instruments contemporains. Paris : Armand Colin.

Rabardel, P. et Pastré, P. (2005). Modèles du sujet pour la conception. Dialectiques activités et développement. Toulouse : Octarès.

Sallaberry, J.-C. (2003). Représentations à bords flous, représentations rationnelles, concepts. In Représentations sociales et formation, Education Permanente. $\mathrm{n}^{\circ} 155$, pp. 7-35.

Scollon, R., Bhatia, V., Li D et Yung, V. (1999). Blurred genres and Fuzzy Identities in Hong-Kong public discourse : Foundational Ethnographic Issues in the Study of Reading. Applied Linguistics. vol. 20, $\mathrm{n}^{\circ} 1$, pp. 22-43.

Traverso, V. (2004). L'analyse des conversations. Paris : Armand Colin.

Weissberg, J. M. (1999). Présences à distance. Paris : L'Harmattan.

Tableau : Analyse a priori des séances sur l'empire de Charlemagne 